\title{
O escore SPADI, idade, nível de escolaridade e gênero são preditivos de autoeficácia em pacientes com dor no ombro?
}

\author{
Are SPADI score, age, level of education, and gender predictive of self-efficacy in patients \\ with shoulder pain?
}
La puntuación SPADI, la edad, el nivel educativo y el género son predictores de autoeficacia en pacientes con dolor de hombro

Natália Borges Agostinho', Júlia Gonzalez Fayão², Jaqueline Martins³, Anamaria Siriani de Oliveira ${ }^{4}$

RESUMO | Fatores intrínsecos, como nível de escolaridade, idade e gênero, se relacionam com dor e disfunção, assim como a autoeficácia pode modificar o efeito que a dor e disfunção têm sobre resultados clínicos dos pacientes. Este estudo transversal investigou se o escore no Shoulder Pain and Disability Index (SPADI), idade, nível de escolaridade e gênero são preditivos de autoeficácia em pacientes com dor no ombro e se há diferença nos níveis de dor e incapacidade entre grupos de idade e gênero. Foram analisados dados sociodemográficos e pontuações do SPADI e do Chronic Pain Self- Efficacy Scale (CPSS) de um banco de dados de pacientes atendidos em um ambulatório de fisioterapia público especializado em ombro, um total de 123 pacientes com média de idade de $54( \pm 11,54)$, SPADI de 67,56 $( \pm 22,54)$ e CPSS de 182,22 $( \pm 61,76)$. A análise de regressão linear múltipla mostrou o SPADI como único fator preditivo de autoeficácia ( $\beta=$ 1,39 [IC95\%=-1,84 a -0,93], $p<0,001)$, explicando 23\% de sua variância $\left(r^{2}=0,23\right)$. A análise de variância (ANOVA) mostrou que a pontuação do SPADI foi significantemente diferente entre gêneros (diferença média=22,27; $p<0,001$ ), mas similar entre grupos de idade (diferença média=7,04; $p=0,16)$. Concluiu-se que os pacientes que se queixaram de dor no ombro em um ambulatório de fisioterapia público especializado em ombro foram a maioria mulheres de meia-idade, que cursaram apenas o ensino fundamental, apresentaram dor e incapacidade importantes e uma alta autoeficácia. Sendo que a pontuação no questionário SPADI foi capaz de prever parcialmente a autoeficácia. Descritores | Autoeficácia; Dor Crônica; Ombro.

ABSTRACT I Factors such as schooling level, age and gender are associated with a more intense pain and a higher level of dysfunction in the shoulder and self-efficacy can modify the effect that pain and dysfunction have on patients' clinical outcomes. Our study investigated if the score on the Shoulder Pain and Disability Index (SPADI), age, schooling level and gender are predictive of selfefficacy in patients with shoulder pain. It also verified if there are differences in levels of pain and disability between age groups and genders. Sociodemographic data and scores from the SPADI and the Chronic Pain Self-Efficacy Scale (CPSS) from a database of patients treated at a public physical therapy clinic specialized in shoulder were analyzed. In total, 123 patients with a mean age of $54( \pm 11.54)$, SPADI of $67.56( \pm 22.54)$ and CPSS of $182.22( \pm 61.76)$ were analyzed. Multiple linear regression analysis showed SPADI as the only predictive factor of self-efficacy $(\beta=-1.39$ [95\% Cl=-1.84 to -0.93$]$, $p<0.001)$, explaining $23 \%$ of its variance $\left(r^{2}=0.23\right)$. ANOVA showed that the SPADI score was significantly different between genders (mean difference=22.27; $p<0.001$ ), but

Este estudo foi realizado no Ambulatório de Reabilitação do Complexo do Ombro (ARCO), inserido na Unidade Básica Distrital de Saúde Sumarezinho Centro Saúde Escola/Dr. Joel Domingos Machado - CSE CUIABÁ, cidade de Ribeirão Preto - SP. Universidade de São Paulo (USP) - Ribeirão Preto (SP), Brasil. E-mail: nataliabagostinho@gmail.com. Orcid: 0000-0002-9192-0157 2Universidade de São Paulo (USP) - Ribeirão Preto (SP), Brasil. E-mail: julia.fayao@gmail.com. Orcid: 0000-0003-3378-7324 3Universidade de São Paulo (USP) - Ribeirão Preto (SP), Brasil. E-mail: jaquelinefisio@usp.br. Orcid: 0000-0002-7601-3209 ${ }^{4}$ Universidade de São Paulo (USP) - Ribeirão Preto (SP), Brasil. E-mail: siriani@fmrp.usp.br. Orcid: 0000-0001-5854-0016 
was similar between age groups (mean difference=7.04, $p=0.16$ ). We concluded that patients that complained of shoulder pain in a public shoulder physical therapy clinic were middle-aged women, who attended only up to middle school, had significant pain and disability, and high self-efficacy. The SPADI score can partially predict self-efficacy.

Keywords | Self Efficacy; Chronic Pain; Shoulder.

RESUMEN I Los factores intrínsecos, como el nivel educativo, la edad y el género, están relacionados con dolor y disfunción, así como la autoeficacia puede modificar el efecto que el dolor y la disfunción tienen en los resultados clínicos de los pacientes. Este estudio transversal tuvo como objetivo evaluar si la puntuación del Índice de discapacidad y dolor de hombro (SPADI), la edad, el nivel educativo y el género son predictores de la autoeficacia en pacientes con dolor de hombro y si existe una diferencia en los niveles de dolor y discapacidad entre grupos de edad y género. Se analizaron los datos sociodemográficos y las puntuaciones de SPADI y la Chronic Pain Self- Efficacy Scale (CPSS) de una base de datos de pacientes que recibieron atención en una clínica pública de fisioterapia especializada en hombro; un total de 123 pacientes con promedio de edad de $54( \pm 11,54)$, SPADI de 67,56 $( \pm 22,54)$ y CPSS de 182,22 ( $\pm 61,76)$. El análisis de regresión lineal múltiple reveló el SPADI como el único predictor de autoeficacia ( $\beta=-1,39$ [IC95\%=-1,84 a -0,93], $p<0,001$ ), lo que explica el 23\% de su varianza $\left(r^{2}=0,23\right)$. El análisis de varianza (Anova) demostró que la puntuación SPADI fue significativamente distinta entre los géneros (diferencia media=22,27; $p<0,001$ ), pero similar entre los grupos de edad (diferencia media=7,04; $p=0,16$ ). Se concluyó que los pacientes que se quejaban de dolor de hombro en una clínica pública de fisioterapia, especializada en hombro, eran en su mayoría mujeres de mediana edad, que tenían cursada la escuela primaria, presentaban dolor y discapacidad significativos y alta autoeficacia. La puntuación en el cuestionario SPADI fue capaz de predecir parcialmente la autoeficacia.

Palabras clave | Autoeficacia; Dolor Crónico; Hombro.

\section{INTRODUÇÃO}

A dor crônica no ombro está entre as queixas musculoesqueléticas altamente prevalentes e que aparece frequentemente na prática clínica do fisioterapeuta ${ }^{1}$. Sintomas relacionados a essa articulação afetam de 7 a $34 \%$ dos adultos em algum momento da vida, compreende, principalmente, a faixa etária de 42 a 55 anos $^{2}$, mulheres ${ }^{2}$ e com nível médio de escolaridade ${ }^{3}$. Essa alta prevalência gera uma quantidade grande de pacientes com limitações funcionais, restrições de participação social, perturbações do sono, sofrimento emocional ${ }^{4}$ e custos ao serviço público ${ }^{5}$.

Diferentes fatores de risco se associam ao desenvolvimento da dor no ombro ${ }^{5}$. A literatura mostra correlações entre dor no ombro e fatores intrínsecos (idade, gênero, escolaridade e peso $)^{2,6}$, fatores relacionados ao trabalho (exposição a movimentos repetitivos, esforços vigorosos, carregamento de peso e utilização de força ${ }^{7}$, e fatores psicossociais (alta demanda mental, baixo suporte social, estresse e depressão) $)^{8}$.

Crenças relacionadas à dor possuem grande influência no desenvolvimento, transição e perpetuação da dor musculoesquelética ${ }^{9}$, assim como na cronificação da dor no ombro ${ }^{10}$. A autoeficácia percebida é uma crença que vem ganhando destaque na literatura e representa a convicção individual de que se pode executar, com algum controle, comportamentos necessários para atingir um determinado resultado ${ }^{11}$. Altos níveis de autoeficácia relativos à saúde determinam o quanto as pessoas se esforçam para desenvolver ou mudar comportamentos que afetam diretamente suas vidas ${ }^{12}$, enquanto baixos níveis de autoeficácia, na avaliação inicial de pacientes com dor no ombro, reduzem o efeito preditivo positivo no tratamento fisioterapêutico de dores menos intensas e incapacidade do ombro, ou seja, a resposta do paciente ao tratamento será inferior ao esperado ${ }^{8}$. Além disso, pacientes com dor crônica e baixa autoeficácia demandam mais atendimentos dos serviços de saúde ${ }^{13}$.

As percepções de autoeficácia podem ser modificadas com intervenções específicas e podem contribuir para a melhoria da disfunção e de sintomas depressivos, e para adesão ao tratamento e tolerância à dor ${ }^{14}$. O nível de autoeficácia de pacientes com dor musculoesquelética crônica no momento da alta da fisioterapia também apresenta relação com uma maior percepção clínica de melhora, menor intensidade de dor e menor número de sessões de fisioterapia ${ }^{15}$. Portanto, é importante que o clínico inclua o nível de autoeficácia referente à dor crônica na avaliação inicial do paciente, e estratégias para aumentar a autoeficácia devem ser consideradas um alvo de intervenção ${ }^{8}$, o que engloba geralmente uma relação terapeuta-paciente que visa mudar as percepções e crenças do paciente por meio da educação, promovendo o automanejo da dor ${ }^{16}$. 
A autoeficácia percebida não é uma medida de desfecho classicamente avaliada pelo clínico, mas tem ganhado atenção no cenário do tratamento da dor crônica no ombro, bem como pouco se sabe da sua correlação com aspectos intrínsecos do paciente e com os níveis de dor e incapacidade autorrelatado pelo mesmo. Assim, este trabalho teve como objetivo primário investigar se o escore no Shoulder Pain and Disability Index (SPADI), idade, nível de escolaridade e gênero são preditivos de autoeficácia em pacientes com dor no ombro. $\mathrm{O}$ objetivo secundário do estudo foi investigar se há diferença nos níveis de dor e incapacidade entre grupos de idade e gênero. A hipótese é de que alguns desses fatores estão associados com a autoeficácia e que o SPADI é diferente para gênero e idade.

\section{METODOLOGIA}

Este estudo observacional transversal analisou um banco de dados de um ambulatório de fisioterapia público especializado em ombro, no período de julho de 2017 a junho de 2019. O estudo seguiu as recomendações do Strengthening the Reporting of Observational Studies in Epidemiology $y^{17} \mathrm{e}$ foi aprovado pelo Comitê de Ética local. Foram coletados $100 \%$ dos registros dos pacientes que se enquadraram nos critérios de inclusão, não havendo perdas por se tratar de coleta de dados armazenados em um banco de dados. O estudo partiu de 167 pacientes, dos quais foram incluídos 123 com idade superior a 18 anos e com queixa principal de dor no ombro relacionada ao espaço subacromial. Foram excluídos os dados de 44 pacientes, com diagnósticos referentes à trauma, como luxações e fraturas, envolvendo o ombro ou o membro superior, e sintomas de acometimento neurológico, como paralisias ou parestesia no membro superior. Analisou-se os dados sociodemográficos autorrelatados (idade, gênero e escolaridade) e as pontuações de dois questionários adaptados e validados para população brasileira, o Shoulder Pain and Disability Index (SPADI) e a Chronic Pain Self- Efficacy Scale (CPSS).

O SPADI é um questionário que avalia dor e incapacidade associadas às disfunções especificamente do ombro. Consiste em 13 itens distribuídos nos domínios de função (8) e dor (5), sendo cada item pontuado em uma escala numérica de 0 a 10 pontos. A pontuação total e por cada domínio é convertida em porcentagem para valores que variam de 0 a 100, com a maior pontuação indicando pior condição de disfunção do ombro. A versão brasileira apresenta confiabilidade (Coeficiente de
Correlação Interclasse=0,94) e consistência interna (Alpha de Cronbach - $\alpha=0,89)$ excelentes da pontuação total e para cada domínio ${ }^{18}$.

A CPSS é uma escala que avalia a percepção de autoeficácia e a capacidade para lidar com as consequências da dor em paciente com dor crônica. Consiste em 22 itens distribuídos nos domínios de controle da dor (5), funcionalidade (9) e controle dos sintomas (8). Cada crença é classificada em uma escala que varia de 10 a 100 e corresponde à certeza que se tem em relação a cada item. A pontuação pode ser total ou para cada domínio. A pontuação mínima é 30 e a máxima 300, com a maior pontuação indicando melhor autoeficácia. A versão brasileira apresenta consistência interna $(\alpha=0,94)$ excelente da pontuação total ${ }^{19}$. Pacientes com dor musculoesquelética que obtiveram pontuação nesse questionário de 172 ou menos foram classificados com baixa autoeficácia e aqueles que pontuaram acima de 172 pontos alta autoeficácia ${ }^{15}$.

Análises estatísticas descritivas simples foram feitas extraindo a média, o desvio-padrão, os números absolutos e a porcentagem dos dados sociodemográficos da classificação da pontuação nos questionários. A análise de variância de duas vias (ANOVA two-way com ajuste de SIDAK) foi realizada para verificar se existe diferença na pontuação do questionário SPADI entre os fatores idade (adultos-jovens foram considerados na faixa etária de 15 a 59 anos e idosos na idade igual ou superior a 60 anos, conforme Política Nacional do Idoso, Lei no ${ }^{\circ}$ 8842, de 4 de janeiro de 1994$)^{20}$ e gênero (feminino e masculino), e também se existe efeito de interação entre eles.

Por fim, realizou-se a análise de regressão linear múltipla no método forward para determinar o grau de influência de fatores preditores nos níveis de autoeficácia percebida. Foram consideradas como variáveis independentes dados contínuos da pontuação total do SPADI e idade, e dados categóricos de escolaridade (nível básico, fundamental, médio ou superior) e gênero (feminino e masculino). Os coeficientes padronizados (Beta) e não padronizados ( $\beta$ ) foram mensurados com a intenção de saber o quão fortemente cada variável preditora influenciava a variável dependente (autoeficácia). O coeficiente Beta foi calculado em unidades de desvio-padrão e o coeficiente $\beta$ em suas unidades naturais. Antes da análise de regressão linear múltipla, foi feita a análise de regressão linear simples para identificar as variáveis com influência significativa (estatística $\mathrm{T}$ do coeficiente $\beta$ ) na autoeficácia ${ }^{21}$. Somente as variáveis com influência significativa foram incluídas na regressão linear múltipla, que contemplou todos os pré-requisitos exigidos. As análises foram realizadas no 
SPSS versão 17 para Windows (SPSS Inc. Chicago, IL) e o nível de significância foi de 0,05 .

\section{RESULTADOS}

Foram analisados dados de 123 pacientes atendidos em um ambulatório de fisioterapia público especializado em ombro. A média de idade foi de 54 anos (desviopadrão=11,54 anos), com predominância do gênero feminino e que cursaram apenas o ensino fundamental. Além disso, a maioria dos pacientes obteve pontuação total da escala CPSS superior a 172 pontos (59,35\%) (Tabela 1).

Tabela 1. Dados sociodemográficos dos pacientes e pontuação nos questionários ( $n=123$ )

\section{Variáveis}

\section{Valores}

Idade, N (\%)

$\begin{array}{ll}60 \text { anos ou mais (idosos) } & 40(32,52) \\ 20 \text { a } 59 \text { anos (adultos) } & 83(67,48) \\ \text { Gênero, N (\%) } & \\ \text { Feminino } & 91(73,98) \\ \text { Masculino } & 32(26,02) \\ \text { Escolaridade, N (\%) } & \\ \text { Analfabeto } & 02(01,63) \\ \text { Fundamental } & 67(54,47) \\ \text { Médio } & 44(35,77) \\ \text { Superior } & 10(08,13)\end{array}$

Shoulder Pain and Disability Index (SPADI), média (DP), [mín; máx]

Domínio de dor

$66,14(22,76)$

$[10,0 ; 100,0]$

Domínio de incapacidade

$54,71(22,25)$

$[2,5 ; 95,0]$

Pontuação Total

$67,56(22,54)$

$[94,0 ; 28,0]$

Chronic Pain Self-Efficacy Scale (CPSS), N (\%)

Indivíduos com pontuação igual ou menor a 172+

$50(40,65)$

Indivíduos com 173 pontos ou mais

$73(59,35)$

Chronic Pain Self-Efficacy Scale (CPSS), média (DP), [mín; máx]

Domínio de controle da dor

$58,46(23,35)$

$[12,0 ; 100,0]$

$68,56(20,17)$,

Domínio de funcionalidade

Domínio de controle dos sintomas

$[10,0 ; 100,0]$

$62,24(25,36)$

$[11,25 ; 100,0]$

$182,22(61,76)$

Pontuação Total

$[45,25 ; 300,0]$

DP: desvio-padrão; mín./máx.: amplitude de valores mínimo e máximo 172 pontos ou menos é um indicativo de baixa autoeficácia em indivíduos com condiçōes musculoesqueléticas crônicas.

A pontuação do SPADI foi significantemente diferente entre gêneros $[\mathrm{F}(1,119)=19,88$; diferença média $=22,27$ (IC95\%=12,38; 32,16), $\mathrm{p}<0,001]$, mas similar entre os grupos de idade $[\mathrm{F}(1,119)=1,99 ;$ diferença média=7,04 (IC95\%=-2,85; 16,93), $\mathrm{p}=0,16]$ e não houve efeito de interação $[\mathrm{F}(1,119)=1,58 ; \mathrm{p}=0,21]$ (Tabela 2).

Tabela 2. Análise de variância do SPADI para os fatores de gênero e idade $(n=123)$

$\begin{array}{lcccc} & \begin{array}{c}\text { Feminino } \\ \text { Média (DP) }\end{array} & \begin{array}{c}\text { Masculino } \\ \text { Média (DP) }\end{array} & \begin{array}{c}\text { Diferença Média } \\ (\text { IC95\%) }\end{array} & \text { P valor } \\ \text { Adulto } & 63,86(21,01) & 47,88(16,75) & 15,99(6,67 ; 25,31) & 0,001^{+} \\ \text {Idoso } & 63,10(19,67) & 34,55(22,68) & 28,55(11,10 ; 45,99) & 0,002^{+} \\ \text {Comparações intragrupo para feminino } & & \\ \text { Adulto (n=57) - Idoso (n=34) } & 0,77(-7,77 ; 9,30) & 0,859 \\ \text { Comparações intragrupo para masculino } & & \\ \text { Adulto (n=26) - Idoso (n=6) } & 13,32(-4,52 ; 31,16) & 0,142\end{array}$

tDiferença entre os gêneros ( $p<0,05)$; $n=$ tamanho da amostra.

A média de pontuação na escala CPSS foi 182,22 $( \pm 61,76)$, e estratificando por idade e gênero obtivemos média de pontuações de $176,97( \pm 63,46)$ para homens adultos ( $\mathrm{n}=26)$; 202,02 $( \pm 43.49)$ para homens idosos $(\mathrm{n}=6) ; 182,45( \pm 66,43)$ para mulheres adultas $(\mathrm{n}=57)$; e $173,74( \pm 67,39)$ para mulheres idosas $(n=34)$.

A regressão linear simples demonstrou significante influência da pontuação do SPADI $(\beta=-1,39 ; p<0,001)$, gênero $(\beta=27,08 ; p=0,03)$ e escolaridade $(\beta=14,31$; $\mathrm{p}=0,09)$ na autoeficácia, com ausência de influência da idade $(\beta=-0,06 ; p=0,90)$. A regressão linear múltipla revelou que a pontuação do SPADI foi a única variável independente capaz de prever a autoeficácia $(\beta=-1,39$ [IC95\%=-1,84 a -0,93], $\mathrm{p}<0,001)$ e explicou $23 \%$ de sua variância $\left(r^{2}=0,23\right)$. A análise resultou em um modelo estatisticamente significativo [ $F(1,121)=36,21 ; p<0,001]$, com Beta=-0,48, no qual autoeficácia $=263,773-1,39 x$ (pontuação do SPADI)].

\section{DISCUSSÃO}

Este estudo caracterizou a incapacidade e a autoeficácia de pacientes atendidos em um ambulatório público de fisioterapia especializado em ombro e identificou a população que se queixa de dor no ombro que é formada por mulheres, na faixa etária dos 50 anos, que cursaram apenas o ensino fundamental, apresentam dor e incapacidade significativa e alta autoeficácia. A análise da diferença na pontuação do questionário SPADI entre os fatores idade e gênero mostrou que as mulheres, independentemente da idade, revelam maior autorrelato de dor e incapacidade do que os homens. A análise de regressão resultou em um 
modelo estatisticamente significativo no qual apenas a pontuação do questionário SPADI explicou a variância da autoeficácia. A idade, o gênero e a escolaridade não foram capazes de prever a autoeficácia.

O SPADI foi capaz de prever a autoeficácia em $23 \%$, ou seja, um quarto de sua variância, e a piora da dor e incapacidade em 1 ponto no SPADI implica na redução da autoeficácia em 1,39 ponto no CPSS. Essa observação é relevante ao clínico, pois indica que pacientes com baixa autoeficácia podem manifestar pior percepção da sua condição clínica, o que corrobora com Souza et al. (2020) ${ }^{15}$ que observaram uma associação moderada entre maior percepção de autoeficácia e melhor percepção de melhora do status clínico no momento da alta da fisioterapia, para pacientes com dor musculoesquelética crônica. As expectativas do paciente em relação à melhora com o tratamento fisioterapêutico e à alta autoeficácia quanto à dor ${ }^{14}$ também já haviam sido indicadas como fatores que influenciam positivamente a pontuação do questionário $\mathrm{SPADI}^{8}$. Além disso, uma revisão sistemática mostrou forte evidência de associação de autoeficácia à adesão de fisioterapia domiciliar, o que pode implicar em uma melhora clínica da dor e função ao final do tratamento.

A literatura tem mostrado que a combinação de autoeficácia com o nível de dor e incapacidade pode conduzir a diferentes desfechos da terapia. Altos níveis de dor e disfunção relacionados à alta autoeficácia reduzem a probabilidade de persistência dos sintomas no ombro ${ }^{17}$. No entanto, a probabilidade de dor persistente no ombro aumenta em indivíduos com baixa pontuação no SPADI e que apresentam menor autoeficácia no momento de admissão na fisioterapia. Sujeitos com baixo nível de intensidade de dor e de escores de autoeficácia têm resultados semelhantes ou piores que aqueles com alto nível de disfunção no ombro e alta autoeficácia ${ }^{8,22}$. Dessa forma, ações que interfiram positivamente na autoeficácia devem ser inseridas na reabilitação a fim de assegurar a aderência ao programa proposto de exercícios, bem como garantir os resultados que seriam esperados para o paciente na ausência de comprometimento dos níveis de autoeficácia.

A idade, o gênero e a escolaridade não foram capazes de prever a autoeficácia, o que ratifica o estudo de Souza et al. ${ }^{15}$ em que não constataram uma relação da autoeficácia com a idade nem com a duração dos sintomas em pacientes com dor musculoesquelética crônica. Futuros estudos precisam investigar outros previsores de autoeficácia, para auxiliar na promoção desse comportamento durante o tratamento de indivíduos com dor no ombro. A literatura apresenta associação da autoeficácia com as emoções, em que altos níveis de "otimismo" e "esperança" se relacionam com menor dor e disfunção musculoesquelética ${ }^{23}$. Portanto, é recomendado à fisioterapia conduzir avaliações que identifiquem esses componentes psicológicos, mas a intervenção pode requerer um olhar mais atento do fisioterapeuta, assim como uma abordagem multiprofissional.

A percepção de autoeficácia pode ser modificada, uma vez que envolve componentes cognitivos e culturalmente adquiridos ${ }^{19}$. Para tanto, são propostos modelos de intervenções cognitivas com o objetivo de intervir em crenças e percepções do paciente por meio da educação ${ }^{16}$, bem como promover o automanejo da dor por meio de um plano de ação com feedback do progresso, estratégias de resolução de problemas, persuasão social, dentre outros. Essencialmente, a educação do paciente envolve a explicação clara da condição, resolução de questões, decisão terapêutica compartilhada, feedback a respeito do desempenho durante a terapia, aconselhamento de promoção e prevenção relacionados à saúde e comprovação da capacidade do profissional ${ }^{16,24}$. Sendo assim, é importante que os profissionais da saúde sejam encorajados a identificar pacientes com níveis reduzidos de autoeficácia e sejam treinados para realizar adequadamente esse tipo de intervenção ${ }^{25}$.

Este estudo tem como pontos positivos o uso de questionários validados para a população brasileira, com excelente consistência interna, caracterizando uma amostra de pacientes que frequentaram um ambulatório de fisioterapia público especializado em ombro. As limitações são a utilização de um banco de dados secundário, a avaliação realizada por diferentes fisioterapeutas e a amostra de um único serviço de atendimento, o que dificulta a generalização dos dados. Neste trabalho, não houve medidas para emoções e fatores de cognição (cinesiofobia e catastrofização), bem como não foram coletadas informações como índice de massa corporal, situação de trabalho e percepção autorrelatada do estado de saúde que podem ser investigadas como preditores de autoeficácia em futuros estudos. Além disso, nosso estudo se restringe a explicar associações e não relações de causa e efeito, assim futuras pesquisas podem incluir o uso de autoeficácia como intervenção adicional, relacionada a desfechos de dor e incapacidade.

\section{CONCLUSÃO}

É possível concluir que a autoeficácia foi prevista pela pontuação do SPADI e não teve relação com fatores intrínsecos de idade e gênero. 


\section{AGRADECIMENTOS}

Nós gostaríamos de agradecer a Coordenação de Aperfeiçoamento de Pessoal de Nível Superior - Brasil (CAPES) pelo auxílio financeiro que possibilitou a execução deste trabalho.

\section{REFERÊNCIAS}

1. Greving K, Dorrestijn O, Winters J, Groenhof F, van der Meer K, Stevens $M$, et al. Incidence, prevalence, and consultation rates of shoulder complaints in general practice. Scand J Rheumatol. 2012;41(2):150-5. doi: 10.3109/03009742.2011.605390

2. Luime JJ, Koes BW, Hendriksen IJM, Burdorf A, Verhagen AP, Miedema HS, et al. Prevalence and incidence of shoulder pain in the general population; a systematic review. Scand J Rheumatol. 2004;33(2):73-81. doi: 10.1080/03009740310004667

3. Dunn WR, Kuhn JE, Sanders R, An Q, Baumgarten KM, Bishop JY, et al. Symptoms of Pain Do Not Correlate with Rotator Cuff Tear Severity. J Bone Jt Surg. 2014;96(10):793-800. doi: 10.2106/JBJS.L.01304

4. Page MJ, O'Connor DA, Malek M, Haas R, Beaton D, Huang H, et al. Patients' experience of shoulder disorders: a systematic review of qualitative studies for the OMERACT Shoulder Core Domain Set. Rheumatology. 2019;58(8):1410-21. doi: 10.1093/ rheumatology/kez046

5. Pribicevic M. The Epidemiology of Shoulder Pain: A Narrative Review of the Literature. In: Ghosh S. Pain in Perspective. London: InTechOpen; 2012. p. 147-86. doi: 10.5772/52931

6. Miranda H, Punnett L, Viikari-Juntura E, Heliövaara M, Knekt P. Physical work and chronic shoulder disorder. Results of a prospective population-based study. Ann Rheum Dis. 2008;67(2):218-23. doi: 10.1136/ard.2007.069419

7. Linaker $\mathrm{CH}$, Walker-Bone K. Shoulder disorders and occupation. Baillieres Best Pract Res, Clin Rheumatol. 2015;29(3):405-23. doi: 10.1016/j.berh.2015.04.001

8. Chester R, Khondoker M, Shepstone L, Lewis JS, Jerosch-Herold C. Self-efficacy and risk of persistent shoulder pain : results of a Classification and Regression Tree (CART) analysis. Br J Sports Med. 2019;53:825-34. doi: 10.1136/bjsports-2018-099450

9. Wertli MM, Rasmussen-Barr E, Held U, Weiser S, Bachmann LM, Brunner F. Fear-avoidance beliefs - A moderator of treatment efficacy in patients with low back pain: a systematic review. Spine J. 2014;14(11):2658-78. doi: 10.1016/j.spinee.2014.02.033

10. Reilingh ML, Kuijpers T, Tanja-Harfterkamp AM, van der Windt DA. Course and prognosis of shoulder symptoms in general practice. Rheumatology. 2008;47(5):724-30. doi: 10.1093/ rheumatology/ken044

11. Bandura A. Autoeficacia: hacia una teoría unificadora del cambio de comportamiento. Psychol Rev. 1977;84(2):191-215. doi: 10.1037/0033-295X.84.2.191
12. Anderson KO, Dowds BN, Pelletz RE, Edwards TW, PeetersAsdourian C. Development and initial validation of a scale to measure self-efficacy beliefs in patients with chronic pain. Pain. 1995;63(1):77-83. doi: 10.1016/0304-3959(95)00021-J

13. Mann EG, Johnson A, Gilron I, Van DenKerkhof EG. Pain management strategies and health care use in communitydwelling individuals living with chronic pain. Pain Med. 2017;18(12):2267-79. doi: 10.1093/pm/pnw341

14. Chester R, Jerosch-Herold C, Lewis J, Shepstone L. Psychological factors are associated with the outcome of physiotherapy for people with shoulder pain: a multicentre longitudinal cohort study. Br J Sports Med. 2018;52(4):269-75. doi: 10.1136/ bjsports-2016-096084

15. Souza CM, Martins J, Libardoni TC, Oliveira AS. Self-efficacy in patients with chronic musculoskeletal conditions discharged from physical therapy service: a cross-sectional study. Musculoskeletal Care. 2020;18(3):365-71. doi: 10.1002/msc.1469

16. Pincus T, Holt N, Vogel S, Underwood M, Savage R, Walsh DA, et al. Cognitive and affective reassurance and patient outcomes in primary care: a systematic review. Pain. 2013;154(11):2407-16. doi: 10.1016/j.pain.2013.07.019

17. Malta M, Cardoso LO, Bastos FI, Magnanini MMF, Silva CMFP. Iniciativa STROBE: subsídios para a comunicação de estudos observacionais. Rev Saude Publica. 2010;44(3):559-65. doi: 10.1590/S0034-89102010000300021

18. Martins J, Napoles BV, Hoffman CB, Oliveira AS. Versão Brasileira do Shoulder Pain and Disability Index: tradução, adaptação cultural e confiabilidade. Braz J Phys Ther. 2010;14(6):527-36. doi: 10.1590/S1413-35552010000600012

19. Salvetti MDG, Pimenta CADM. Validação da Chronic Pain Selfefficacy Scale para a língua Portuguesa. Arch Clin Psychiatry. 2005;32(4):202-10. doi: 10.1590/s0101-60832005000400002

20. Miranda GMD, Mendes ACG, Silva ALA. O Envelhecimento populacional brasileiro: desafios e consequências sociais atuais e futuras. Rev Bras Geriatr Gerontol. 2016;19(3):507-19. doi: 10.1590/1809-98232016019.150140

21. Domholdt E. Physical Therapy Research: Principles and Applications. 2nd ed. Philadelphia: Saunders; 2000.

22. Struyf F, Geraets J, Noten S, Meeus M, Nijs J. A multivariable prediction model for the chronification of non-traumatic shoulder pain: A systematic review. Pain Physician. 2016;19(2):1-10.

23. De Baets L, Matheve T, Meeus M, Struyf F, Timmermans A. The influence of cognitions, emotions and behavioral factors on treatment outcomes in musculoskeletal shoulder pain: a systematic review. Clin Rehabil. 2019;33(6):980-91. doi: 10.1177/0269215519831056

24. Ashford S, Edmunds J, French DP. What is the best way to change self-efficacy to promote lifestyle and recreational physical activity? A systematic review with meta-analysis. Br J Health Psychol. 2010;15(2):265-88. doi: 10.1348/135910709X461752

25. Martinez-Calderon J, Zamora-Campos C, Navarro-Ledesma S, Luque-Suarez A. The Role of Self-Efficacy on the Prognosis of Chronic Musculoskeletal Pain: a Systematic Review. J Pain. 2018;19(1):10-34. doi: 10.1016/j.jpain.2017.08.008 